\title{
Image Acquisition Method of Kiwifruit Picking Robot
}

\author{
Shuai Su, Longsheng Fu, Fanian Zhang, Yongjie \\ Cui* \\ College of Mechanical and Electronic Engineering, \\ Northwest A\&F University, \\ Yangling, Shannxi, 712100 China \\ *e-mail: cuiyongjie@nwsuaf.edu.cn
}

\author{
Shuai Su \\ Beijing Research Center of Intelligent Equipment for \\ Agriculture \\ Beijing, China \\ shuai1117@139.com
}

\begin{abstract}
In order to overcome the complex and diverse background in kiwifruit image that acquired by approximate level capture method (ALCM), a look up capture method (LUCM) to acquire fruit images was proposed. According to study fruit identification process and effect of the kiwifruit in the image acquired by LUCM, and compared to that of ALCM, following conclusions could be obtained: (1) the LUCM could reduce background noises effectively, (2) the LUCM overcame that target fruit overlapping to each other in the image of the ALCM and achieved precise positioning, (3) the LUCM could determine the picking sequence of target fruit in the image in one-time. However, the LUCM is sensitive to light condition which is going to be studied by using artificial light in the future.
\end{abstract}

Keywords-kiwifruit; image acquisition; identification; picking sequence;

\section{INTRODUCTION}

China is the largest country for cultivating kiwifruit. And Shaanxi is the largest plantation province in China, its production accounts for about $70 \%$ in China, and more than $33 \%$ in the world [1]. Until the end of 2011, the cultivation area of kiwifruit in Shaanxi was around $4.72 \times 10^{4} \mathrm{hm}^{2}$, and the production was reached $7.357 \times 10^{5}$ t, which made Shaanxi became the largest production area in the world [2]. However, harvesting of kiwifruit in this area is still mainly relying on manual picking which is labor-intensive. Therefore, it is necessary to do research on picking robot of kiwifruit.

Kiwifruit is generally use scaffolding style cultivation methods, and its fruit color is similar to color of background, such as hay, leaves, stems, and stalk. Besides, some fruit are adjacent to each other, and some fruit are blocked by leaves. Therefore, it is necessary to accurately segment, recognize, and extract features of kiwifruit under natural environment for developing the kiwifruit picking robot vision system.

For the fruit recognition under natural environment, related researches include cucumber which has similar color to the background and was detected using near infrared spectroscopy [3-4], fruits that are adjacent and overlapped to each other but have quite different color to background, such as tomato [5-6], apple [7-8], citrus [9], and lychee [10]. For kiwifruit recognition, Ding et al. used R-B color parameters to segment it, but they didn't identify each single fruit [11]. Cui et al. studied the method for fruit recognition and feature extraction based on the color and shape features of kiwifruit in nature, extracted fruit features as centroid coordinates, long axis end coordinates, long axis length and short axis length, and reached a success rate to $89.1 \%$ [12]. However, fruit images which acquired by approximate level capture method (ALCM) in this study include complex and diverse background. These backgrounds would cause a large number of noises in image segmentation, and thus influence the accuracy of segmentation and recognition.

Therefore, according to the scaffolding cultivation method and growth characteristics of kiwifruit, this paper proposed a look up capture method (LUCM) to acquire fruit images. By comparing with the recognition effect of ALCM, the fruit recognition process and its effect was studied for fruit images acquired by the LUCM.

\section{MATERIAls AND MethodS}

\section{A. Image Acquistion}

Fruit images of the most common cultivar group of kiwifruit ('Hayward') were collected by a digital camera (Sony DSC-S700) at the Meixian Kiwifruit Experimental Station of Northwest A\&F University, Shaanxi Province, China. Image acquisition was carried out under sunlight in some sunny days from October to November, 2012. For ALCM, the camera is located near to the underside of the scaffolding, and its central axis is approximately parallel to the horizontal plane of the scaffolding. For LUCM, the camera is located below the scaffolding, and its central axis is vertical to the horizontal plane of the scaffolding. The camera is set around $1.2 \mathrm{~m}$ from target fruit. Images taken in this distance contains 4-7 fruit that recognizable, and its resolution is 640 pixels $\times 480$ pixels in the jpg format.

\section{B. Identification of Kiwifruit}

To improve success rate of kiwifruit recognition, a method for fruit recognition and feature extraction based on the color and shape features of kiwifruit in nature was studied by Cui et al [12-13]. It could reduce the influences of complicated background, different kiwi growth state and natural lighting condition. First, $R-G$ color component was chosen by comparing different color spaces. Then the optimum partition coefficient of $n R-G$ color characteristics was determined according to the image evaluation method of error segmentation pixel, and $0.9 R-G$ was selected finally. The Otsu method was used for threshold segmentation and morphological operation was employed to remove residual 
noise, and then the regions of target fruits and backgrounds were successfully separated. The image boundary was extracted by Canny operator and consequent elliptic Hough transform, which made the target fruit be recognized separately. Also, fruit features as centroid coordinates, long axis end coordinates, long axis length and short axis length were extracted. Results show that this method is effective in the recognition of kiwifruit. And thus it is adapted in this study to recognize target fruit in the kiwifruit images that acquired in different capture method. The fruit recognition flow is shown in Fig. 1, and the recognition process is shown in Fig. 2.

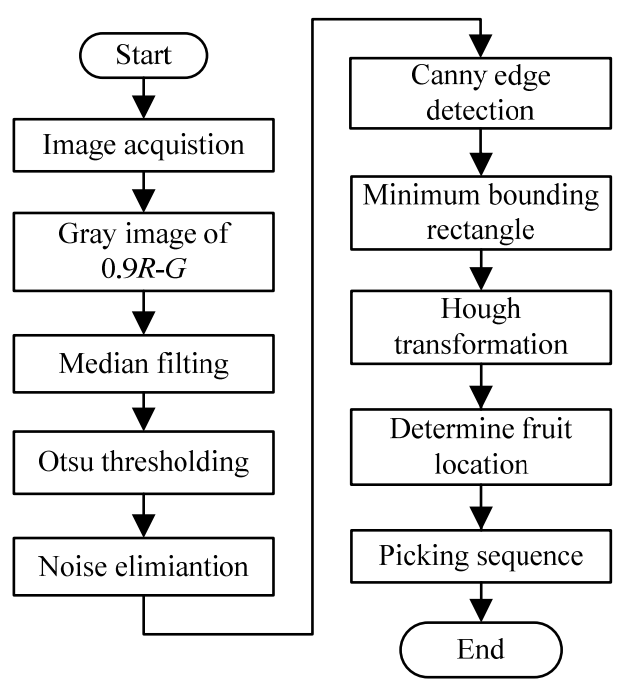

FIGURE 1. RECOGNITION FLOW OF KIWIFRUIT

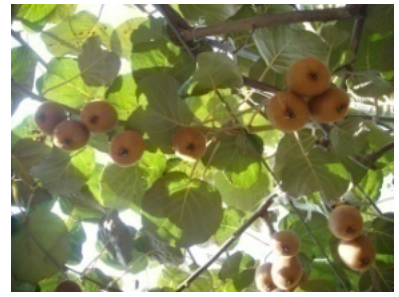

(a)

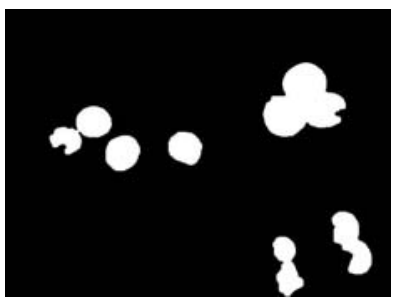

(e)

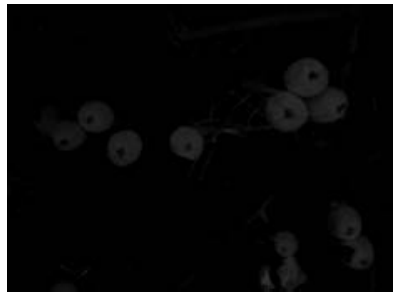

(b)

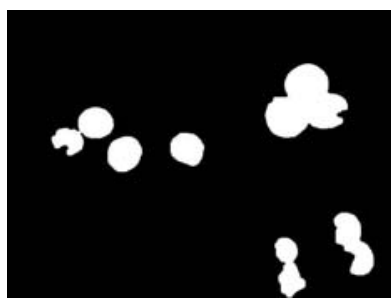

(f)

\section{RESULTS AND DISCUSSION}

Comparing with the fruit recognition process of kiwifruit image that collected by ALCM as shown in Fig. 3, the LUCM has following advantages:

\section{A. Few Background Noise}

The image acquired by ALCM contained complex background, including weed and other materials on the ground, as shown in Fig. 3(a). Those foreign materials caused a large number of noises in the image segmentation, as shown in Fig. 3(c). In addition, lots of non-target fruit in small size was also included in the image of ALCM since its great scene depth. It caused huge interference to the identification of closed target fruit. Moreover, fruit are hanging under the branches since the scaffolding cultivation method of kiwifruit, they are blocked by some fall down branches and leaves in the image. All of that would eventually affect the fruit segmentation and correct identification.

However, for image acquired by LUCM, there were few target fruit are obscured by leaves and less effect from background noises such as weeds, stalk, and leaves in uneven illumination which normally happened in the image acquired by ALCM, as shown in Fig. 2(a).

\section{B. Fruit located exactly}

Kiwifruit is grown clustered, and its height is substantially same. Therefore, the target fruit were overlapped to each other in the image acquired by ALCM, as shown in Fig 3 (a). In that case, the accuracy of Hough transform on the target fruit was affected by the behind fruit, and thus it is difficult to determine the precise location of the target fruit, as shown in Fig. 3(h). Moreover, the accuracy of positioning will directly have an influence on the manipulator control and picking efficiency.

While for the image acquired by the LUCM, all the target

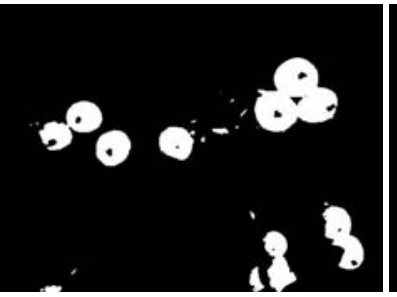

(c)

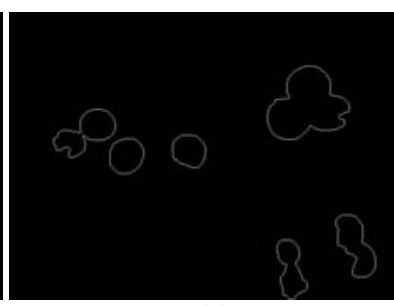

(g)

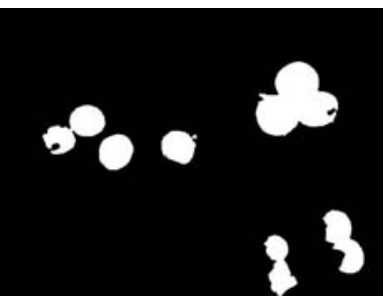

(d)

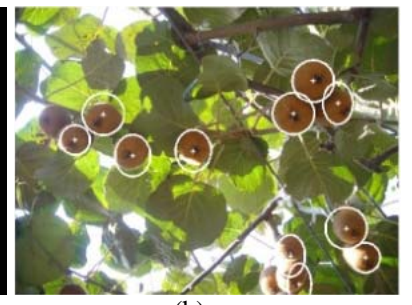

(h)

FIGURE 2. FRUIT IDENTIFICATION PROCESS OF KIWIFRUIT IN THE IMAGE ACQUIRED BY LUCM

(A) ORIginal IMAGE; (B) GRAY IMAGE AFTER 0.9R-G; (C) BINARY IMAGE AFTER 0.9R-G; (D) ELIMINATION OF SMALL AREA NOISES; (E) MORPHOLOGICAL OPERATOR; (F) ELIMINATION OF SMALL AREA; (G) CANNY EDGE DETECTION; (H) HOUGH TRANSFORMATION 


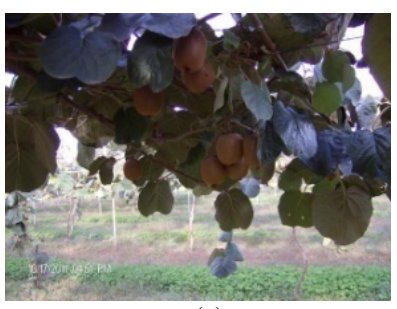

(a)

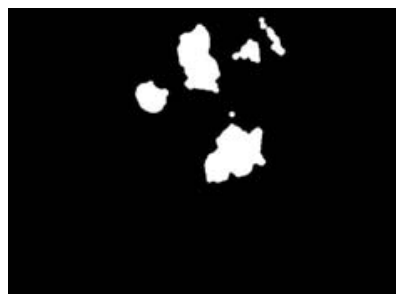

(e)

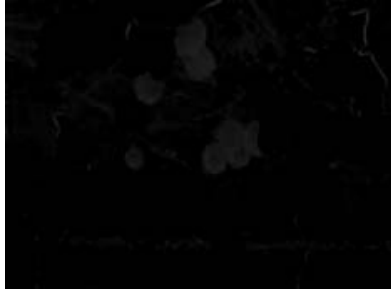

(b)

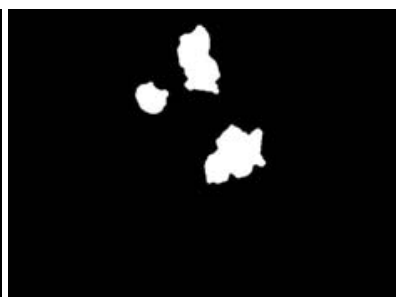

(f)

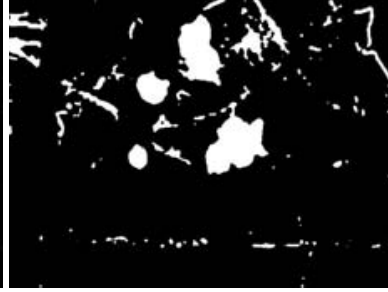

(c)

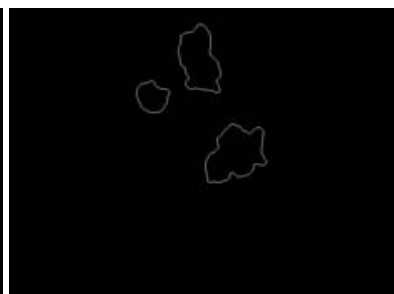

(g)

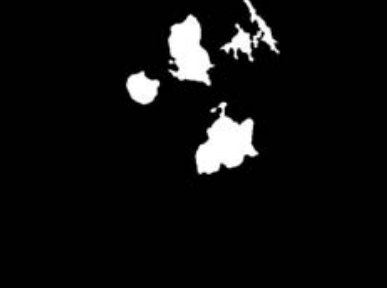

(d)

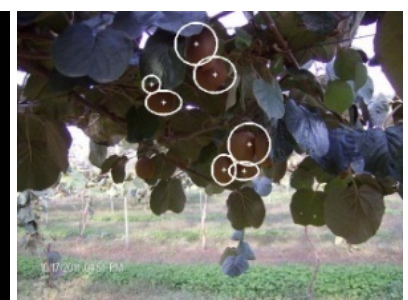

(h)

FIGURE 3. FRUIT IDENTIFICATION PROCESS OF KIWIFRUIT IN THE IMAGE ACQUIRED BY ALCM

(A) ORIGINAL IMAGE; (B) GRAY IMAGE AFTER 0.9R-G; (C) BINARY IMAGE AFTER 0.9R-G; (D) ELIMINATION OF SMALL AREA NOISES; (E) MORPHOLOGICAL OPERATOR; (F) ELIMINATION OF SMALL AREA; (G) CANNY EDGE DETECTION; (H) HOUGH TRANSFORMATION

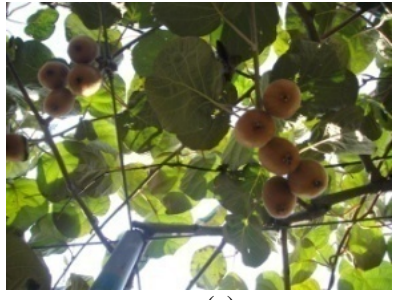

(a)

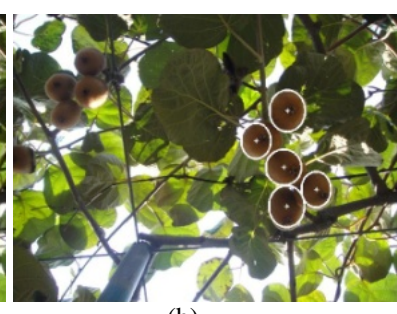

(b)
FIGURE 3. FRUIT IDENTIFIED BADLY IN THE IMAGE ACQUIRED BY LUCM (A) ORIGINAL IMAGE; (B) IMAGE AFTER IDENTIFICATION

fruit almost on the one plane and no overlapping because of the growth style of kiwifruit, as shown in Fig. 2(a). In that condition, the outline each single target fruit could be obtained exactly, and thus the location of target fruit could be determined correctly by using image processing.

\section{Determine picking sequence in one-time}

Because the target fruit were overlapped to each other in the image acquired by ALCM, the picking robot has to recognize and locate the most external fruit and pick it firstly, and then turn to the internal fruit. It cannot determine picking sequence at firstly in one-time, and thus the efficiency is very low. While the target fruit were on the same plane in the image acquired by LUCM, the picking robot could determine picking sequence in one-time by calculating the coordinates of the centroid of each target fruit. And then transfer the positions of all the fruit to its control part and pick the fruit sequentially. Therefore, the picking efficiency and accuracy could be improved.

Further, in order to facilitate the identification of each single target fruit by characteristic parameters of fruit shape, its area in the image needs to be stable in a certain range. In the LUCM, it could be achieved by setting the distance between lens and the target fruit, and thus fixed mostly the fruit size in the image. It is very helpful to the subsequent identification of each individual target fruit by the shape parameters. According to field survey and experiment, the optimal distance between lens and the target fruit was set to $1 \mathrm{~m}$. In that distance, there are normally 4-7 recognizable target fruit included in the image.

However, the LUCM was strongly influenced by the light since most of them were blocked by leaves, as shown in Figure 4. For example, a cluster of fruit (including 4) in the upper left corner of Fig 4(a) weren't recognized since insufficient illumination, as shown in Fig. 4(b). It will be studied by using artificial light to solve it in the future.

\section{CONCLUSIONS}

According to research the fruit identification of the kiwifruit in the image acquired by LUCM, and compared to that of ALCM, following conclusions could be acquired: (1) the LUCM could reduce background noises effectively, (2) the LUCM overcame the target fruit overlapped to each other in the image of the ALCM and achieved precise positioning, (3) the LUCM could determine the picking sequence of target fruit in image in one-time. However, the LUCM is sensitive to light condition which is going to be studied by using artificial light in the future.

\section{REFERENCES}

[1] Z. Sun, "Fruit area and yield was increased 12 years in Shannxi," China Fruit News, vol. 30, pp. 44-45, 2013.

[2] J. Wang, L. Yi, Q. Bai, and M. Guo, "Study on Risk Zoning of High Temperature and Drought Disaster for Kiwifruit in Shaanxi," Chinese Agricultural Science Bulletin, vol. 29(7), pp. 105-110, 2013.

[3] T. Yuan, J. Zhang, and W. Li, "Feature acquisition of cucumber fruit in unstructured environment using machine vision," Transactions of the Chinese Society for Agricultural Machinery, vol. 40(8), pp. 170174, 2009. 
[4] E.J. Henten, B.J. Tuijl, and G.J. Hoogakker, “An autonomous robot for de-leafing cucumber plants grown in a high wire cultivation system,” Biosystems Engineering, vol. 94(3), pp. 317-323, 2006.

[5] A. Arman, M.M. Asad, and M. Kaveh, "Recognition and localization of ripen tomato based on machine vision,” AJCS, vol. 5(10), pp. 1144-1149, 2011.

[6] R. Xiang, Y. Ying, and H. Jiang, "Recognition of overlapping tomatoes based on edge curvature analysis," Transactions of the Chinese Society for Agricultural Machinery, vol. 43(3), pp. 180-183, 2012.

[7] J.P. Wachs, H.I. Stern, and T. Burks, "Low and high-level visual feature-based apple detection from multi-modal images,” Precision Agriculture, vol. 11(10), pp. 717-735, 2010.

[8] J. Rakuna, D. Stajnkoa, and D. Zazulab, "Detecting fruits in natural scenes by using spatial-frequency based texture analysis and multiview geometry," Computers and Electronics in Agriculture, vol. 76(1), pp. 80-88, 2011.
[9] J. Cai, X. Zhou, and Y. Li, "Recognition of mature oranges in natural scene based on machine vision," Transactions of the CSAE, vol. 24(1), pp. 175-178, 2008.

[10] J. Xiong, X. Zou, L. Chen, and A. Guo, "Recognition of mature litchi in natural environment based on machine vision," Transactions of the Chinese Society for Agricultural Machinery, vol. 42(9), pp. 162-166, 2011.

[11] Y. Ding, N. Geng, and Q. Zhou, "Research on the object extraction of kiwifruit based on images," Control \& Automation, vol. 25(18), pp. 294-295, 2009.

[12] Y. Cui, S. Su, X. Wang, Y. Tian, P. Li, and F. Zhang, "Recognition and Feature Extraction of Kiwifruit in Natural Environment Based on Machine Vision," Transactions of the Chinese Society for Agricultural Machinery, vol. 44(5), pp. 247-252, 2013.

[13] Y. Cui, S. Su, Z. Lv, P. Li, and X. Ding, "A method for separation of kiwifruit adjacent fruits based on Hough transformation," Journal of Agricultural Mechanization Research, vol. 34(12), pp. 166-169, 2012. 